\title{
Modulation of the gut microbiota represents a new management for non-alcoholic fatty liver disease
}

\author{
Farnush Bakhshimoghaddam ${ }^{1}$, Mohammad Alizadeh ${ }^{2}$ \\ ${ }^{1}$ Student Research Committee, Department of Nutrition, ${ }^{2}$ Department of Nutrition, Food and Beverages Safety Research Center, Faculty of \\ Medicine, Urmia University of Medical Sciences, Urmia, Iran \\ Correspondence to: Mohammad Alizadeh. Department of Nutrition, Food and Beverages Safety Research Center, Faculty of Medicine, Urmia \\ University of Medical Sciences, Serow Highway, Nazloo, 5756115111 Urmia, Iran. Email: alizade85@yahoo.com; alizadeh.m@umsu.ac.ir. \\ Provenance and Peer Review: This article was commissioned by the Editorial Office, Hepatobiliary Surgery and Nutrition. The article did not undergo \\ external peer review. \\ Comment on: Sharpton SR, Maraj B, Harding-Theobald E, et al. Gut microbiome-targeted therapies in nonalcoholic fatty liver disease: a systematic \\ review, meta-analysis, and meta-regression. Am J Clin Nutr 2019;110:139-49.
}

Submitted Sep 05, 2019. Accepted for publication Oct 08, 2019.

doi: $10.21037 /$ hbsn.2019.10.01

View this article at: http://dx.doi.org/10.21037/hbsn.2019.10.01

With the worldwide spread of diet westernization, sedentary lifestyle, and obesity, non-alcoholic fatty liver disease (NAFLD) has becoming frequent disorder. NAFLD is the presence of hepatic fat infiltration more than $5 \%$ of liver cells, in lack of alcohol abuse or steatogenic drugs. NAFLD includes a spectrum of liver disorder, ranging from steatosis and non-alcoholic steatohepatitis (NASH) to fibrosis, cirrhosis and hepatocellular carcinoma (1). Metabolic syndrome and systemic micro-inflammation are the most relevant metabolic disorders related to this spectrum of diseases (2). There is currently no approved pharmacological treatment for NAFLD. The management strategy of NAFLD involves lifestyle interventions, including diet, exercise, and behavior modification (1).

The intestinal microbiota has been a topic of scientific research for a recent decade. The gut microbiota composed of organized community of microorganisms (3). The literature to date suggests points to a critical impact of the intestinal flora and intestinal barrier on the pathogenesis of NAFLD and other metabolic disorders (4). Dysbiosis, which is defined as changes in intestinal bacteria, and small intestine bacterial overgrowth, which is related to dietary habits, genetic predisposition, and physical inactivity can influence energy homeostasis. Dysbiosis, impaired intestinal barrier function, and adipose tissue related inflammation act directly through increased translocation of bacterial endotoxins or lipotoxins, or indirectly, through the regulation of insulin sensitivity and resulting liver steatosis (5). The interplay between gut microbiota and NAFLD onset is summarized and illustrated in Figure 1. Previous studies documented that severity of steatosis in NAFLD patients correlated with small bowel bacterial overgrowth and increased intestinal permeability (6). The novel progresses in knowledge about the human gut microbiota have changed our sight of disease and treatment. Therapeutic manipulations with prebiotics, probiotics, and synbiotic as microbiome-based interventions are potential targets for NAFLD management via modulating the enteric flora (Figure 2). Considering the ease of use of these supplements, their safety and low cost, they beneficial impact on hepatic steatosis and fibrosis $(7,8)$; oral probiotics are live microorganisms that have potentially benefits for the host through its effects in the intestinal tract if consumed in adequate amounts (9). Prebiotics are fermentable fibers that facilitate the growth of probiotics and thereby benefit health (10). Synbiotics which are combination of prebiotics and probiotics have a potentially stronger effect in modulating the gut microbiota than either probiotics or prebiotics alone (11). A meta-analysis has reported probiotic supplementation can improve liver enzymes, serum cholesterol, and insulin sensitivity in patients with NAFLD (12). Evidence of a meta-analysis declare that probiotic and synbiotic supplementation may improve lipid profile, glycemic variable, liver enzymes, and inflammatory mediator by alternating the type and/or function of the intestinal microbiota (11). In our previous 


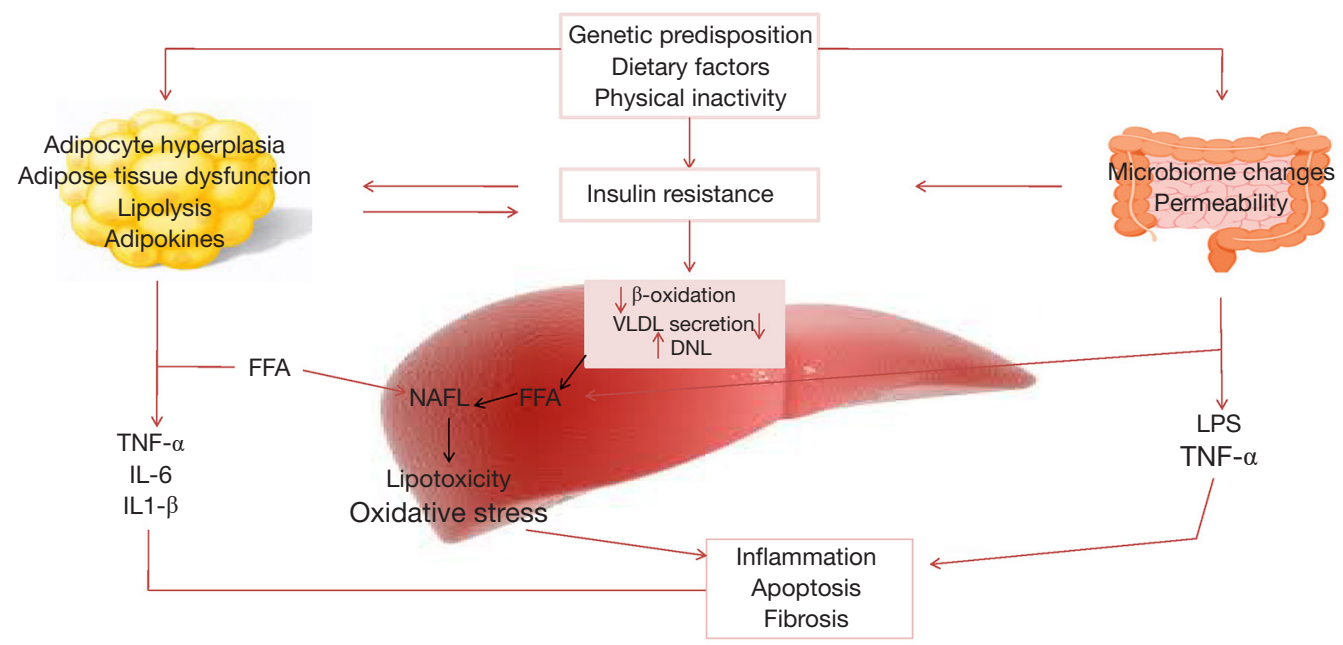

Figure 1 The gut microbiota and non-alcoholic fatty liver disease. Obesity, dysbiosis of gut microbiota and increased intestinal permeability leads to insulin resistance. The inflammatory mediators predispose to development of non-alcoholic fatty liver disease. DNL, de novo lipogenesis; FFA, free fatty acid; IL-1 $\beta$, interleukin-1 $\beta$; IL-6, interleukin-6; LPS, lipopolysaccharides; NAFL, non-alcoholic fatty liver; NAFLD, non-alcoholic fatty liver disease; NASH, non-alcoholic steatohepatitis; TNF- $\alpha$, tumor necrosis factor- $\alpha$.

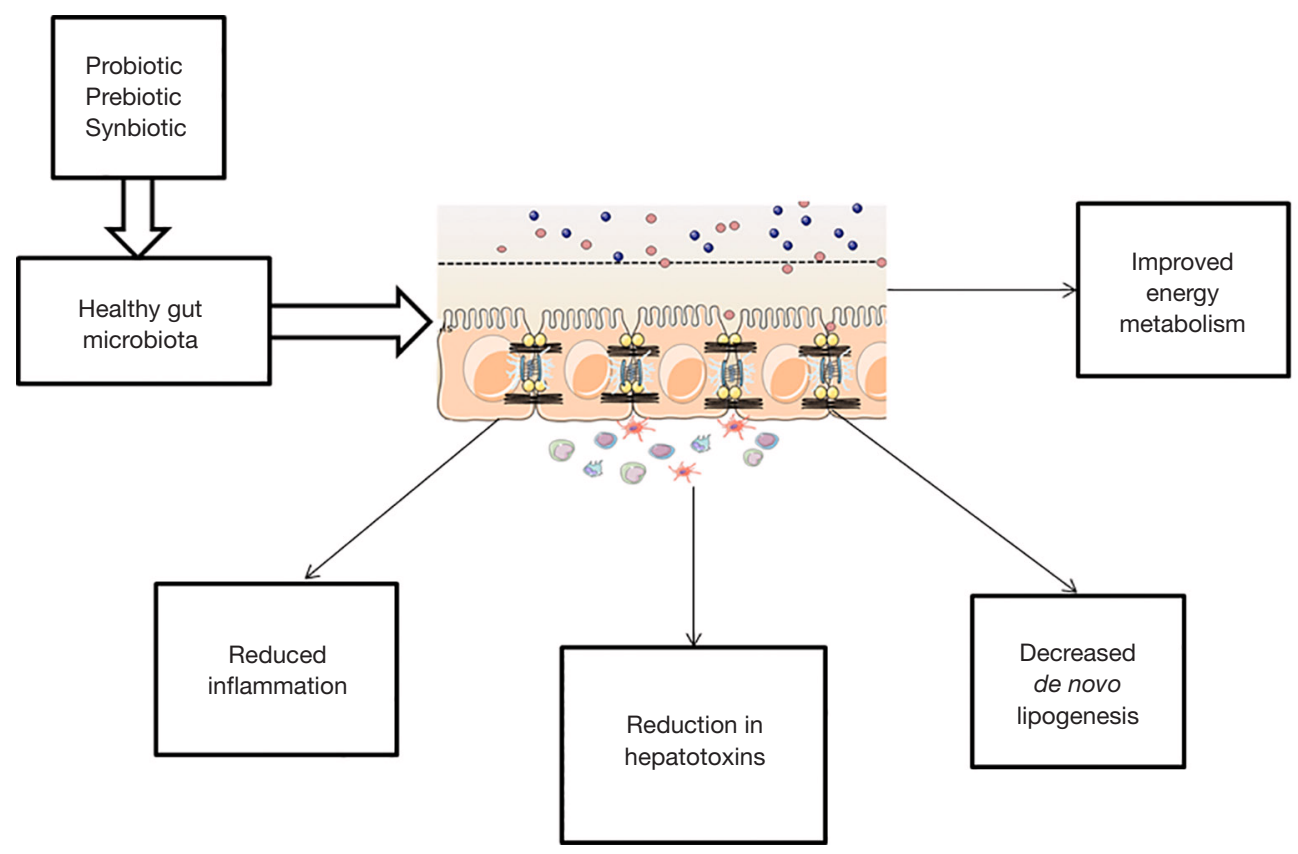

Figure 2 Potential mechanisms of action for synbiotics, prebiotics, and probiotics on non-alcoholic fatty liver disease. Synbiotics, prebiotics, and probiotics could improve gut epithelial barrier, reduction in inflammation, bacteria-derived hepatotoxins, energy metabolism, and de novo lipogenesis.

study, we found that synbiotic supplementation along with lifestyle modification has beneficial effect on hepatic echogenicity, liver enzymes, lipoprotein and glycemic profile, oxidants and antioxidants biomarkers, and adipokine and gut peptide levels. To our knowledge, this was the first randomized clinical trial that showed that synbiotic yogurt increased glucagon-like peptide- 2 as the intestinal permeability index and $\mathrm{C} 1 \mathrm{q} / \mathrm{TNF}$-related protein 5 as 
regulation of lipid and glucose marker (13).

Meta-analysis conducted by Sharpton et al., in the current issue of Am 7 Clin Nutr (14), provides a rich data set from associations between synbiotics/probiotics supplementation and decrease of liver stiffness, steatosis and inflammation in NAFLD. The diagnosis of NAFLD was established according to magnetic resonance imaging, ultrasound, or elastography. The investigators included 1,252 patients from 21 randomized clinical trials which investigated probiotics and synbiotics in comparison to control, with intervention duration ranging from 2 to 7 months. In this study primary outcomes were serum alanine aminotransferase concentration, and hepatic fibrosis and steatosis measured by elastography and ultrasound, respectively. Also secondary outcomes were change in body mass index, triglyceride, and homeostatic model of insulin resistance. The analysis by Sharpton et al. showed that synbiotics/probiotics were related to significant reduction in hepatic steatosis and fibrosis and serum alanine aminotransferase concentration. Uses of synbiotic/ probiotic decreased body mass index only in probiotic trials. In both groups, probiotic and synbiotic did not effect on triglyceride. Also, the authors did not find the beneficial effect from synbiotics/probiotics on homeostatic model of insulin resistance. In summary, the study presented by Sharpton et al. suggested modulation of gut microbiota with multibiotics-based interventions to promote the growth of good microbiota, may ameliorate dysbiosis in patients with NAFLD and improve liver-specific and metabolic outcomes. As the authors point out, synbiotics or probiotics supplementations through modification of the intestinal microbiota could promise a new management approach in patients with NAFLD.

Gut microbiome-targeted therapies are direct and feasible approach against the harmful effects of dysbiosis on the human host. Therefore, the intestinal microbiota has appeared as an attractive target for potential therapeutics in metabolic diseases (4). In recent years, our knowledge regarding the functions and composition of the intestinal microflora has markedly increased; however, these are still in their infancy and need further development and testing. To a great extent, this has been attributed to the progression of genomic analyses of microbial communities, which have recognized the critical portion of the microbiota to human health or diseases (15).

\section{Acknowledgments}

Funding: None.

\section{Footnote}

Conflicts of Interest: All authors have completed the ICMJE uniform disclosure form (available at https://hbsn. amegroups.com/article/view/10.21037/hbsn.2019.10.01/coif). The authors have no conflicts of interest to declare.

Ethical Statement: The authors are accountable for all aspects of the work in ensuring that questions related to the accuracy or integrity of any part of the work are appropriately investigated and resolved.

Open Access Statement: This is an Open Access article distributed in accordance with the Creative Commons Attribution-NonCommercial-NoDerivs 4.0 International License (CC BY-NC-ND 4.0), which permits the noncommercial replication and distribution of the article with the strict proviso that no changes or edits are made and the original work is properly cited (including links to both the formal publication through the relevant DOI and the license). See: https://creativecommons.org/licenses/by-nc$\mathrm{nd} / 4.0 /$.

\section{References}

1. Chalasani N, Younossi Z, Lavine JE, et al. The diagnosis and management of nonalcoholic fatty liver disease: practice guidance from the American Association for the Study of Liver Diseases. Hepatology 2018;67:328-57.

2. Gao X, Fan JG. Diagnosis and management of nonalcoholic fatty liver disease and related metabolic disorders: Consensus statement from the Study Group of Liver and Metabolism, Chinese Society of Endocrinology. J Diabetes 2013;5:406-15.

3. Tandon P, Garcia-Tsao G. Bacterial infections, sepsis, and multiorgan failure in cirrhosis. Semin Liver Dis 2008;28:26-42.

4. Boulange CL, Neves AL, Chilloux J, et al. Impact of the gut microbiota on inflammation, obesity, and metabolic disease. Genome Med 2016;8:42.

5. Miele L, Valenza V, La Torre G, et al. Increased intestinal permeability and tight junction alterations in nonalcoholic 
fatty liver disease. Hepatology 2009;49:1877-87.

6. Volynets V, Kuper MA, Strahl S, et al. Nutrition, intestinal permeability, and blood ethanol levels are altered in patients with nonalcoholic fatty liver disease (NAFLD). Dig Dis Sci 2012;57:1932-41.

7. Li Z, Yang S, Lin H, et al. Probiotics and antibodies to TNF inhibit inflammatory activity and improve nonalcoholic fatty liver disease. Hepatology 2003;37:343-50.

8. Mencarelli A, Cipriani S, Renga B, et al. VSL\# 3 resets insulin signaling and protects against NASH and atherosclerosis in a model of genetic dyslipidemia and intestinal inflammation. PloS One 2012;7:e45425.

9. Nardone G, Rocco A. Probiotics: a potential target for the prevention and treatment of steatohepatitis. J Clin Gastroenterol 2004;38:S121-2.

10. Roberfroid M, Gibson GR, Hoyles L, et al. Prebiotic effects: metabolic and health benefits. Br J Nutr 2010;104 Suppl 2:S1-63.

Cite this article as: Bakhshimoghaddam F, Alizadeh M. Modulation of the gut microbiota represents a new management for non-alcoholic fatty liver disease. HepatoBiliary Surg Nutr 2020;9(2):223-226. doi: 10.21037/hbsn.2019.10.01
11. Khalesi S, Johnson DW, Campbell K, et al. Effect of probiotics and synbiotics consumption on serum concentrations of liver function test enzymes: a systematic review and meta-analysis. Eur J Nutr 2018;57:2037-53.

12. Ma YY, Li L, Yu CH, et al. Effects of probiotics on nonalcoholic fatty liver disease: a meta-analysis. World J Gastroenterol 2013;19:6911-8.

13. Bakhshimoghaddam F, Shateri K, Sina M, et al. Daily consumption of synbiotic yogurt decreases liver steatosis in patients with nonalcoholic fatty liver disease: a randomized controlled clinical trial. J Nutr 2018;148:1276-84.

14. Sharpton SR, Maraj B, Harding-Theobald E, et al. Gut microbiome-targeted therapies in nonalcoholic fatty liver disease: a systematic review, meta-analysis, and metaregression. Am J Clin Nutr 2019;110:139-49.

15. Kong L, Lu Y, Zhang S, et al. Role of nutrition, gene polymorphism, and gut microbiota in non-alcoholic fatty liver disease. Discov Med 2017;24:95-106. 